\title{
La educación física chilena y su profesorado: proponiendo algunos retos para la investigación en el área Chilean physical education and its teachers: proposal of some challenges for research about the subject
}

Alberto Moreno Doña*, Carolina Poblete Gálvez**

*Pontificia Universidad Católica de Valparaíso (Chile), ${ }^{* *}$ Universidad de Santiago de Chile(Chile)

Resumen. El siguiente artículo presenta una caracterización de la educación física chilena a partir de las creencias del profesorado del área sobre la asignatura en cuestión y su relación con la transformación social. Para dicha caracterización se han considerado, también, los cambios que la asignatura ha sufrido a raíz de la implementación de nuevas políticas públicas. A partir de grupos focales, entrevistas en profundidad y la observación participante, como técnicas de recogida de datos, se profundiza en cómo la educación física de carácter tecnocrático sigue siendo el eje vertebrador tanto del decir como del hacer de los profesionales del área. A partir de los datos mostrados, se concluye presentando una serie de retos en y para la investigación en la educación física.

Palabras clave. educación física; transformación social; creencias del profesorado; investigación en educación física.

Abstract. The following paper shows a characterization of Chilean physical education based on the beliefs of teachers in this area about the PE subject and its relationship with social transformation. For this characterization, the changes that the physical education subject has undergone with the implementation of new public policies were also taken into account. Using focal groups, in-depth interviews and participating observation, as well as data collection techniques, the articles explores how a physical education subject with a technocratic character is still the axis on which physical education teachers base their work. Finally, from the data shown, several physical education research challenges are suggested.

Key words. physical education; social transformation; teachers' beliefs; physical education research.

\section{Introducción}

La educación física chilena (en adelante EFCh) está experimentando grandes e importantes cambios en los últimos años. El aumento de dos a cuatro horas semanales de clases para la asignatura en la enseñanza básica (Decreto № 2960/2012), la creación de un sistema de medición de su calidad (SIMCE) (ACE, 2013), la construcción de estándares de desempeño para la formación de profesores en el área (CPEIP, 2013), la creación de una nueva asignatura denominada «Educación Física y Salud» (en adelante EFSA) que sustituye a la Educación Física existente hasta el año 2012 (MINEDUC, 2013) y que propone una estructura curricular basada en competencias, no en objetivos y contenidos, para la construcción de hábitos de actividad física saludable, tanto en enseñanza básica como en media, y la fundación del Ministerio del Deporte en el año 2013 (Ministerio Secretaria General de Gobierno, 2013) son ejemplos claros de lo que podría caracterizarse como una situación ideal para una asignatura que forma parte del currículum escolar. Si a esto le unimos el diseño e implementación de un programa social («Elige Vivir Sano») que pretende dar respuesta a los preocupantes datos existentes en relación a la 'salud' de la población infantil y juvenil en Chile, con altos índices de obesidad y sobrepeso (MINSAL, 2009-2010: 99), la escasa adherencia a la realización de actividad física de manera regular (Ibídem,277) y los malos hábitos nutricionales (Ibídem, 229), sin duda que la primera lectura de lo expresado nos conduce a realizar una evaluación positiva de las intenciones de los gobiernos de turno para con el bienestar de los niños y jóvenes chilenos, además de considerar que se está enalteciendo la asignatura de Educación Física (Educación Física y Salud en la actualidad) como área curricular determinante en la contribución al bienestar de las personas.

Sin embargo, esta situación se constituye en un problema cuando dichos cambios no están basados en evidencias científicas y pedagógicas construidas a partir de una investigación contextualizada al ámbito nacional. Sobre todo si la investigación que se ha generado no ha sido capaz de mostrar la situación actual en la que se encuentra la asignatura. Si nos hacemos eco del cuestionamiento internacional existente sobre la importancia que tienen las políticas públicas basadas en evidencias, no en ocurrencias, (Flecha \& Molina, 2013; Rodríguez \& Flecha, 2011; Ministerio de Educación, 2011), entonces las transformaciones que han tenido lugar en la EFCh se constituyen en un problema, más que en la solución a ciertos inconvenientes sociales y educativos.

En un primer análisis crítico de algunas de las propuestas y cambios

Fecha recepción: 20-12-14- Fecha envío revisores: 20-12-14- Fecha de aceptación: 18-03-15 Alberto Moreno Doña alberto.moreno@ucv.c descritos anteriormente para la EFCh (Moreno, 2011-2014) se observó que el SIMCE en la EFCh es poco coherente con las bases curriculares y los planes y programas de la asignatura. El aumento de horas de la asignatura está basado en una relación, equivocada a nuestro entender, que entiende que más horas es mejor, sin tener claro si lo que se estaba haciendo era bueno, pues no siempre se cumple la relación de que 'más es mejor'. Si no fuera así (existen escasas evidencias al respecto), estaríamos proponiendo más horas de una actividad que no genera aquello que se quiere lograr: la construcción de hábitos saludables (Moreno, Rivera,\& Trigueros, 2014).

Sin duda que desde la perspectiva del colectivo docente, todas las transformaciones sufridas por la EFCh han sido una excelente noticia, pero es difícil estar de acuerdo en que son la mejor manera de contribuir a la solución de un problema tan complejo como la construcción de hábitos saludables entre la población infantil y juvenil. La creación de la nueva asignatura «Educación Física y Salud», si bien plantea cambios relevantes desde el punto de vista curricular, sigue poniendo el énfasis en la realización acrítica de actividad física desde un enfoque biomédico (Moreno, Gamboa,\& Poblete, 2014), ello a pesar de que las escasas investigaciones chilenas existentes son críticas con este modelo (Almonacid \& Matus, 2012; Moreno, et al., 2013; Toro, 2007). Críticas que surgen desde la comprensión de que en aquellos lugares donde la EFCh ha contribuido a la generación de ciertos hábitos saludables entre los escolares es, entre otros motivos, porque ha conseguido transformar la asignatura de la que hablamos en una parte más del entramado social que debe actuar para la construcción de los hábitos mencionados.

No más de cinco proyectos de investigación concursables, desde el año 2005, se han dedicado a investigar alguna problemática relacionada con la EFCh (CONICYT, 2005-2012). Y sólo existen siete revistas científico técnicas en el área, ninguna de ellas indexadas en las tres bases de datos científicas de mayor consideración e importancia en Chile (WOS, SCOPUS y SCIELO). Sin duda que esto último es resultado de un escaso desarrollo científico de la EFCh, pero también es motivo de preocupación al no tener una serie de evidencias científicas y pedagógicas contextualizadas al ámbito nacional que den sustento al desarrollo profesional de todos aquellos que se dedican a la asignatura. Por último, unido a lo anterior y como consecuencia de ello, entre otros factores, el estatus social de la EFCh es bastante cuestionable. Desde los años 80, y a raíz de los cambios administrativos que llevaron a que el colectivo docente comenzara a desarrollar su trabajo en condiciones laborales deterioradas (Daroch, 2010), el prestigio social del profesorado ha sufrido una importante merma (Tenti, 2005). Dicha desvalorización social ha sido aún más fuerte entre los profesionales dedicados a la EFCh, causado, entre otros motivos, por el escaso valor educativo asociado a las prácticas corporales en comparación con las intelectuales (Kirk, 1990, 2006, 2007) y la equivocada dicotomía entre trabajo (dimensión 
intelectual) y descanso (juego y deporte) (Loaiza, Sánchez, \& Villegas, 2004).

Es más, sumado a las escasas evidencias científicas que sustenten las transformaciones relatadas, estas se han llevado a cabo sin la consideración de lo que el profesorado del área que trabaja en los diferentes centros educativos piensa, dice y hace (Moreno, et al., 2013). Es por ello que desde hace algunos años venimos investigando las creencias del profesorado y su quehacer pedagógico en relación a cómo la asignatura puede contribuir, o está contribuyendo, a uno de los graves problemas que acechan a la sociedad chilena, la desigualdad de oportunidades, pues consideramos que la misma afecta las posibilidades de construcción de una educación física anclada y sustentada en la justicia y la equidad.

Es desde aquí que una serie de preguntas han dirigido nuestro trabajo investigativo en los últimos años, y nos han permitido establecer una serie de cuestionamientos y objetivos que desarrollamos en este trabajo, a saber:

Preguntas de investigación:

¿Cuáles son las creencias de los docentes de EF respecto a la función social que debe aportar el área?

¿Qué barreras a nivel personal, profesional e institucional perciben para orientar la EF hacia un enfoque pedagógico que busque la transformación social y trate de minimizar las desigualdad?

¿Qué soluciones o alternativas ven viables los docentes de EF de incorporar en el área para generar un trabajo transformativo?

¿Cómo se estructuran, temporal y espacialmentelas, clases de EF?

¿Cómo actúan los profesores/as ante las minorías presentes en sus estudiantes?

¿Qué aprendizajes y contenidos se muestran con mayor regularidad en las sesiones de clase?

A partir de estas preguntas, proponemos los siguientes objetivos de investigación:

Analizar las creencias docentes en relación a la función social de la EFCh.

Identificar los impedimentos, en diversas dimensiones del quehacer profesional, para hacer de la EF una asignatura que contribuya ala disminución de las desigualdades de origen.

Describir las soluciones que el mismo colectivo docente propone para la generación de un trabajo transformativo en el área.

Comprender las características de las clases de educación física.

\section{Metodología}

Nuestra lógica metodológica ha estado centrada en la comprensión crítica de las creencias del profesorado de educación física en relación a la desigualdad social-educativa y las posibilidades de que la educación física pueda aportar en paliar las desigualdades de origen. Entendemos por desigualdades de origen a aquellas que están relacionadadas con una desigualdad que se da desde el momento del nacimiento y que es consecuencia de una estructura social que las permite y legitima. Hemos optado por una lógica investigativa anclada en el paradigma interpretativofenomenológico a partir del que creemos es posible inferir y comprender dichos significados (Canales, 2006). Desde este enfoque paradigmático, la metodología cualitativa nos permite una comprensión intersubjetiva que emerge a partir de toda la información analizada, entendiendo que la misma esconde un contenido caracterizado, implícitay/o explícitamente, por intencionalidades educativas, finalidades, concepciones, exclusiones, valoraciones, etc.

La metodología utilizada posee un carácter descriptivo, relacional, interpretativo y crítico. Esta elección realizada se basa, principalmente, en las preguntas que nos han dirigido durante el proceso investigativo, dado que nuestra intención ha sido identificar y comprender cualidades y características y no registrar cantidades y frecuencias de aparición de un determinado fenómeno. Para la descripción, interpretación y comprensión de cualidades hemos utilizado conceptos y relaciones entre ellos (Strauss \& Corbin, 2002; Gibbs, 2012).
Las técnicas de recogida de datos utilizadas durante estos años han estado centradas en grupos focales, entrevistas en profundidad y la observación participante. Se realizaron 3 grupos focales y 12 entrevistas en profundidad de carácter individual. La selección de los participantes para los grupos focales (11 participantes en el primero, 8 en el segundo y 6 en el tercero), las entrevistas y las observaciones se realizaron a partir de criterios que permitieron representatividad en función de: (a) de diferentes zonas del país (norte, centro y sur), (b) experiencias pedagógicas en la enseñanza básica y media, (c) sexo, (d) institución donde los participantes se formaron como profesores y profesoras de EF, y (e) tipo de centro educativo donde lleva a cabo su docencia. Tanto las entrevistas como los grupos focales duraron un máximo de 60 minutos. En total se realizaron 100 horas de observación directa entre los tres establecimientos educativos seleccionados.

En los grupos focales hemos indagado en las actitudes y reacciones de un grupo social específico frente a un asunto social o político, o bien un tema de interés como un producto, servicio, concepto, publicidad, idea, etc. Las preguntas son respondidas por la interacción del grupo en una dinámica en que los participantes se sienten cómodos y libres de hablar y comentar sus opiniones.

La entrevista en profundidad tiene como propósito que el investigador se sitúe en el lugar del entrevistado, suponiendo que la realidad de los otros es significativa, cognoscible y explícita (Flick, 2004).

La observación participante es la principal técnica etnográfica de recogida de datos. En ella el investigador pasa todo el tiempo posible con los sujetos que estudia. Refleja sus interacciones y actividades en notas de campo que toma en el momento o inmediatamente después de producirse los fenómenos (Goetz \& LeCompte, 1988). La idea central que nos ha guiado mediante esta técnica es atender a la dimensión del hacer en relación a las percepciones del profesorado sobre las funciones de la EF Chy más específicamente sobre el aporte de la de la misma a la transformación de las desigualdades de origen.

El material ha sido sometido a análisis de contenido apoyado en el programa computacional NVivo 9.0. Los procedimientos de análisis que se han empleado, en general se ciñen a las propuestas de fragmentación y articulación de la «Grounded Theory» (Strauss \&Corbin, 2002). Se realizó codificación abierta, con categorías emergentes; codificación axial, en torno a la agrupación de las categorías emergentes en familias de categorías; y codificación selectiva, mediante un trabajo interpretativo que condujo a la elaboración de un modelo interpretativo.

\section{Resultados}

En este apartado mostraremos los principales hallazgos resultantes de nuestro trabajo investigativo. Sin duda que los años de investigación nos han permitido tener muchos más resultados que los mostrados, pero el espacio limitado que poseemos nos ha llevado a utilizar el criterio de mayor relevancia (frecuencia de aparición en los análisis realizados) para seleccionar lo que a continuación detallaremos. Hemos dividido este apartado en cuatro subapartados. En el primero exponemos las creencias del profesorado en relación a la EFCh, revelando el pensamiento de los docentes en relación a la perspectiva de la EF más presente en sus discursos. Seguidamente nos adentramos en otro subapartado relacionado con las dificultades que el mismo colectivo docente señala para la puesta en práctica de una EF menos reproductora y más transformadora de la realidad educativa y social presente en el país. Continuamos dando cuenta de una caracterización descriptiva de las prácticas docentes. Y finalizamos el apartado explicitando algunas ideas propuestas por el mismo profesorado para la construcción de una EFCh que sea capaz de trabajar por y para la disminución de las desigualdades con las que los niños/as ingresan al sistema escolar.

A lo largo de este apartado podrán encontrar expresiones como GF1, GF2 y GF3. Cuando ello ocurra debe entenderse grupo focal 1, grupo focal 2 y grupo focal 3. También aparecen nombres propios relacionados con los participantes en la investigación. Lógicamente son inventados para salvaguardar el anonimato de los mismos. 


\section{Creencias del profesorado en relación a la EFCh: la reproduc- ción acrítica de una educación físicatecnocrática}

Los docentes hacen alusión constantementeal deporte, la condición física y a un mayor y mejor estado de salud como contenidos centrales de la asignatura. Esta percepción parte de la creencia de que el deporte es sinónimo de mejora de la condición física y que ésta equivale a un mejor bienestar. Sin embargo, esta creencia contradice la evidencia científica que, desde hace décadas, enfatiza que es una lógica equivocada, sobre en todo en niños y niñas, pues en esas edadeslos niveles de condición física están determinados por factores genéticos y de maduración, más que por hábitos de actividad física (Fox,1991). Son los buenos programas de actividad física los que pueden contribuir, entre otras actividades, al bienestar de los niños y niñas. Pero cuando se hace una relación directa y casi exclsuvia entre deporte, mejora de la condición física y bienestar estamos cayendo en un error y debemos ser cuidadosos en esa relación directa, y acrítica, que muchos docentes establecen.

La presencia constante del deporte como contenido casi exclusivo de la EFCh es posible visualizarla en las propias palabras del profesorado: «[...] el objetivo personal que tenemos todos los profesores de EF es la formación en el área del deporte.» (GF1)

Esta idea también se refleja a nivel particular entre los participantes:

«De quinto a segundo de secundaria es un día acondicionamiento y el otro de deportes, y en tercero y cuarto como tienen una vez a la semana este año es solamente deporte, no hay acondicionamiento (...), y de primero a cuarto básico se hace deporte los dos días a la semana». (Adolphe)

Elvira comenta que «[...] la única forma que tú [sic] mueves a los chicos en el colegio es con deporte»

A su vez, la preocupación con el desarrollo de la salud y concretamente, en relación a la lucha contra la obesidad, se hace patente en otro de los grupos focales:

«Hoy en día también hay un problema general y grandote que es la obesidad, entonces nosotros le vamos a mostrar todos los deportes para que después en la vida cotidiana de ellos puedan rescatar uno de toda esa gama de deportes, por algo tenemos atletismo, basquetbol, fútbol, no sé tenemos todos los deportes y eso es nuestro trabajo» Esta relación entre deporte y salud queda aún más clara si rescatamos las palabras de Barack, al decirnos que «[...] la actividad física te da más posibilidades si centras la EF en el rendimiento motor a través de la utilización de tu cuerpo» (GF2).

Los estereotipos son otro eje importante de las creencias del profesorado. El primer estereotipo desarrollado por varios participantes está relacionado con entender que la EF puede ayudar en la formación deportiva y que podría posibilitar que algún estudiante llegara a ser deportista profesional. Evans, por ejemplo, nos señala que «[...] yo siempre les doy ejemplos de deportistas que hoy día son famosos y a [los alumnos] les motiva mucho llegar en algún momento a ser eso. Yo les enseño que pueden perfectamente llegar a ser eso[...]»

El segundo estereotipo se focaliza en la relación acrítica entre la realización de actividad física y la adquisición de ciertos valores que, en última instancia pueden conducir a la superación de la pobreza. «Sí, es súper importante para la parte social el desarrollo de las habilidades que se desarrollan [a través de] la actividad física y el deporte. El espíritu de superación [que puede llevar a] salir de la pobreza es súper importante» (Elvira).

Por último encontramos la presencia de estereotipos de género, lo que dificulta un trabajo pedagógico coeducativo en las clases de EF. Alison considera «[...] que las niñas deben trabajar aparte de los hombres», pues deben «desarrollar otro tipo de cosas».

\section{Dificultades para una práctica pedagógica transformadora}

Las barreras que los participantes señalan para la puesta en práctica real de una EF crítica y constructora de mayor equidad y justicia social son cuatro:
(1) Barreras por la situación curricular de la EF:Aquí se plantean las incoherencias de una lógica curricular que, por un lado, dice poseer una intencionalidad educativa mientras que, por otro lado, se ajusta a, y perpetúa, un sistema de medición de la calidad (SIMCE) centrado en otras intencionalidades (medición del nivel de condición física principalmente), lo que termina constituyendo una barrera para el desarrollo educativo de la EF como asignatura del currículum escolar. «EI SIMCE no mide nada, no mide el estado de salud de un alumno, al contrario, lo inhabilita o lo perjudica. Hicieron el SIMCE y varios alumnos salieron con lesiones lumbares y abdominales» (Jimmy).

(2) Barreras de formación profesional: Se incluyen aquí las referencias a la débil formación que hoy día está recibiendo el profesorado de $\mathrm{EF}$, lo que termina provocando que los mismos docentes no sean capaces de construir una asignatura más ligada al ámbito educativo y no relacionada, únicamente, con la práctica deportiva. Lo mismo ocurre con la formación continua del profesorado. »Es muy importante que nos podamos capacitar. Yo trabajo en un colegio municipal y la capacitación es bastante escasa, los tiempos de capacitación bastante escasos y, en general, las horas de trabajo son muchas. Un profesor que tenga 44 horas va a trabajar 60 y va a llegar a su casa; ¿va a seguir trabajando?» (Evans). Se hace referencia a la complejidad propia de la asignatura en un mundo que ha cambiado excesivamente y que ha provocado que «[...] las enfermedades de la tercera edad aparezcan en la niñez» (Luke), lo que provoca que «trabajemos con niños y jóvenes en una situación para la cual no fuimos formados» (GF2).

(3) Desvalorización social de la EFCh. Es «triste llegar a final de año frustrado. Pasó el año, y me encuentro sometido por un sistema escolarque no me representa en nada [...]. Los apoderados se oponen a mi criterio, el jefe no me apoya y me pide que cambie la planificación [...]. Ello porque la EF no es una disciplina fuerte que no se valora socialmente» (Cornelio).

\section{El quehacer docente en los patios escolares}

Dicho quehacer lo hemos caracterizado a partir de 4 ejes vertebradores de la práctica pedagógica. Existen otros ejes propios de la categorización realizada y que nos han permitido describir, más profundamente, el quehacer docente. Los cuatro que aquí se muestran son los que han mostrado mayor nivel de presencia en las clases analizadas.

Prácticas basadas en el orden y control. Son prácticas docentes autoritarias. Preocupación constante por mantener el silencio, uso de silbato, amenazas y preocupación excesiva por la apariencia. »Oiga. . . eh... van a empezar a cerrar la boquita..., ya que se tiene que escuchar la voz del profesor, no la de ustedes» (Registro de campo).

Prácticas pedagógicas como sesiones de entrenamiento cuyo principal propósito es el rendimiento motor, en detrimento del componente educativo. («se bebe agua sólo al terminar la actividad» / «cuánto más se sufre mejor» / «como los deportistas de élite y los militares) (Registro de campo).

Estilo metodológico excesivamente directivo (uso casi exclusivo del mando directo) donde el profesorado indica lo que hay que hacer y el alumnado ejecuta las órdenes propuestas. En este sentido cabe destacar que «los estilos directivos reproducen todo un entramado de relaciones de poder entre el profesorado y el estudiantado, caracterizadas por el disciplinamiento, la normalización, la competenciay la homogenización» (Blandón, Molina \&Vergara, 2000: 87), aspectos que conducen a generar marcos de violencia y rechazo ante el modelo impuesto por el profesorado.

El proceso calificativo presenta un excesivo énfasis como eje articulador de la práctica pedagógica. Existe una presión constante sobre el alumnado, una presión relacionada con el cuándo y cómo van a ser calificados.

\section{Algunas ideas para el cambio expuestas por el profesorado novel}

Es entre el profesorado de EF novel donde se presenta una fuerte contradicción cuando ingresan al sistema escolar. Dicha contradicción 
viene dada por un fuerte interés caracterizado por las ganas de generar un cambio de carácter cualitativo y las lógicas que, según ellos mismos, siguen los docentes experimentados. La caracterización del cambio tiene como principio fundamental el instaurar la reflexión como mecanismo orientador que invite, incentive y se responsabilice por la amplitud de ella, que debiera abordar diferentes dimensiones: desde «la reflexión del aula, la disciplina», hasta dimensiones más amplias y contextuales como sus «componentes ontológicos, epistemológicos», a la vez que aspectos concretos como «los metodológicos».

Marcos invita a una reflexión epistemológica a partir de la cual entendamos que cuerpo y mente no son dos realidades diferenciadas, pues ello es uno de los estigmas de nuestra área de conocimientos (GF2). Fabiola cree posible la reconstrucción de significados y cuestionamiento de las prácticas tradicionales a través de procesos de reflexión compartidos; esto podría contribuir a la transformación y mejora social. Rocío sueña con los posibles caminos a seguir en el trabajo pedagógico con estudiantes universitarios, principalmente en los primeros años de formación, para lo cual se hace indispensable la consecuencia profesional y la minuciosa articulación entre teoría y práctica (GF1). La actividad física transformadora comienza por la transformación de la persona, en toda su complejidad, y por el cuerpo del participante, pues desde él se posibilita aprender más de uno mismo y de su entorno. Dos cosas son fundamentales, (...) que la actividad física sea una actividad transformadora en la integridad de la persona, cómo desde mi cuerpo aprendo más de mí, cómo desde mi motricidad mejoro mi identidad y a partir de eso me conozco (Mabel). El cuerpo se convierte para Mabel en el centro del trabajo pedagógico, pues según ella los seres humanos escribimos el libro de nuestro cuerpo y en él encontramos la experiencia humana vivida, todo lo tenemos ahí, la violencia, la dulzura (...).

Para operacionalizar estas experiencias en el quehacer pedagógico se hace necesaria una práctica más reflexionada, lo que sin duda requiere de normas acordadas entre los diferentes actores del quehacer educativo (Mabel).

Discusión: nuevos retos para la investigación en la educación física chilena

A partir de todo lo expuesto, consideramos que se hace necesario e indispensable un trabajo científico que nos permita construir una mirada general y compleja sobre el fenómeno de la EFCh. Ello nos invita a plantear algunos retos investigativos para el área curricular en cuestión.

Se hace relevante examinar el estatus social de la EFCh. El estatus de la EFCh está relacionado con la capacidad que dicha asignatura ha tenido para legitimarse en los diferentes contextos donde se practica. Tal es la dimensión del problema, que en algunos países han surgido voces que proponen la eliminación de la EF como parte del currículum escolar (Giesecke, 1998; Lenzen, 2000; Pedraz, 2007). Esta deslegitimación pareciera ser observada por los mismos profesores de EF cuando argumentan que diariamente tienen que vivir situaciones como: suspensión de clases para reforzar otras materias, bajo reconocimiento por parte de algunos directores o colegas de otras asignaturas, desconocimiento de la importancia de la EFCh por parte de los padres y apoderados, y que en muchas escuelas la EFCh es impartida por profesores que no son especialistas y algunos colegas se limitan a entregar un balón para que sus alumnos/as jueguen toda la clase (Moreno et. al., 2013).

La situación comentada en el párrafo anterior ha sido explicada, a partir de numerosas investigaciones (Cárcamo, 2012; Deutscher Sportbund, 2006; Eurydice, 2013; Hardman, 2008; Hardman\& Marshall, 2000; López, et. al., 2005) atendiendo a dos tipos de factores: externos (epistemológicos, políticos, científicos, administrativos y de infraestructura) e internos (quehacer docente en los patios y aulas escolares). A pesar de ello, la investigación de esta problemática ha sido escasamente trabajada en el contexto chileno.

Otro reto relevante para la investigación en EF está relacionado con la Didáctica de la especialidad. No hay lugar a dudas de que el concepto de calidad es polisémico (Santos, 2003) y que ello ha dificultado definir con precisión el significado de ‘calidad de la educación física’. El actual sistema de calidad de la educación física (SIMCE en educación física) confunde calidad del área con desarrollo de la condición física, sin atender a los elementos didácticos que constituyen las clases de la asignatura (Moreno, 2014). Debido a ello se hace necesario caracterizar y analizar críticamente la coherencia entre el quehacer docente (los procesos didácticos que se construyen) y los postulados emanados del Ministerio de Educación. Es por ello que vamos a entender que la didáctica de la EFCh debe ser capaz de orientarse «a los problemas de sedentarismo en el país» (MINEDUC, 2013a: 162) aportando «al desarrollo integral de los estudiantes (...) adquiriendo hábitos de vida saludables (...) y que estos hábitos se constituyan en parte relevante de la cotidianeidad de los estudiantes (...), contribuyendo al bienestar cognitivo, emocional, físico y social del alumno» (Ídem). Es relevante considerar que el Ministerio de Educación plantea un trabajo didáctico orientado a la construcción de hábitos transferibles a la vida cotidiana del alumnado, lo que en el ámbito concreto de la didáctica tiene consecuencias tangibles, a saber: a) orientar las sesiones de EF hacia la autonomía (autogestión) en la realización de las diferentes prácticas corporales (Lorente, 2005); b) pasar del activismo físico a prácticas corporales que incluyan la reflexión sobre la práctica (Gómez \& Prat, 2009); c) desarrollo de una competencia crítica a partir de la cual poder analizar aquellas prácticas corporales que más y menos aportan al bienestar humano y los condicionantes sociales y contextuales que determinan las mismas (Tirado \& Ventura, 2009: Mercedes, 2009; Córdoba, 2011); y d) trabajar por la responsabilidad personal y social que implica la realización de actividades físicas (Escartí, Pascual, \& Gutiérrez, 2005; Escartí, et al., 2009).

Por último, se hace relevante atender a la producción científica en el área de la EFCh. A nivel internacional las revistas se han convertido en el instrumento de comunicación científica fundamental, dando a conocer los resultados más relevantes logrados por los investigadores y retroalimentando la actividad científica producida por los diversos equipos de investigación (Valenciano, Villamón, \& Devís, 2008). Qué se publica, quiénes lo hacen y la forma que adoptan los instrumentos donde se publica (las revistas) son un reflejo de la calidad científica de lo que se produce en un área disciplinar determinada (Delgado, Ruiz, \& Jiménez, 2006). Esto, a su vez, contribuye a la percepción que los diferentes entes sociales construyen en relación a un área en particular, favoreciendo la construcción de un círculo virtuoso: mayor calidad de las publicaciones periódicas genera, entre otras cosas: a) mayor calidad de la ciencia que se realiza; b) mejor estatus social de la disciplina; c) mayores exigencias para esa disciplina y mejor preparación de los profesionales que trabajan en ella. De ahíla relevancia de que la investigación en la EFCh se adentre en la evaluación de sus revistas científico-técnicas.

En el ámbito chileno es prácticamente inexistente el trabajo en esta área, aunque muy recientemente fue terminada, leída y aprobada una tesis doctoral titulada «La construcción del conocimiento histórico de la educación física en Chile. Una mirada a la primera revista latinoamericana en la especialidad durante los años comprendidos entre 1934 y 1962». Si bien esta tesis se ha constituido en un importante aporte al conocimiento de la producción científica en elárea de la educación física, su alcance es limitado, pues se centra en una sola revista y a un periodo de tiempo determinado. A pesar de ello, los resultados obtenidos revelan el nivel de importancia de analizar la producción científica y académica a lo largo de los años, argumentando, con mayores bases contextuales, la forma en que se han construido las identidades colectivas en esta disciplina (Poblete, 2013). También es muy relevante mencionar el proyecto de investigación financiado por el Fondo Nacional de Investigación Científica y Tecnológica de Chile(Ref. 2013-n¹1130187), aún en desarrollo, cuya autoría corresponde al Dr. Mikel Pérez Gutiérrez de la Universidad Autónoma de Chile. El título de dicho proyecto es «Análisis bibliométrico e interpretación sociocultural de la producción científico-técnica en elámbito de las Ciencias de laActividad Física y del Deporte en Chile».

Por último, es importante resaltar algunos otros retos que se derivan del trabajo de investigación realizado. A saber:

Necesidad y urgencia por profundizar en el ámbito de la forma- 
ción permanente del profesorado de educación física.

Comparar el pensamiento del profesorado novel con el del profesor con mayor experiencia. Ello permitiría comprender cómo ha evolucionado y/o involucionado dicho pensamiento.

Contrastar el pensamiento del profesorado sobre la educación física con el pensamiento de otros actores educativos: alumnado, profesorado de otras áreas curriculares, etc.

\section{Conclusiones y aplicaciones}

Para finalizar este trabajo se han generado algunas conclusiones.

(1) En el discurso que se ha ido instalando en la educación física chilena y sus profesores es posible visualizar ciertos principios que orientan la asignatura y el quehacer docente hacia la construcción de una educación física alternativa, más democrática, reflexiva y orientada a un proceso educativo capaz de trabajar la integralidad del ser humano. Aunque es relevante mencionar que este discurso es minoritario y es por ello que el desarrollo del mismo en este trabajo es poco visible. A pesar de ello, la perspectiva de mayor presencia en la EF y su profesorado está relacionada con una visión tecnocrática y reproductora de la EF escolar. Una perspectiva ligada casi exclusivamente al deporte, el desarrollo de la condición física y la reproducción de ciertos estereotipos sociales.

(2) La realidad mostrada, una entre otras posibles, puede ser de utilidad para que el profesorado del área oriente su quehacer reflexivo hacia una transformación de las prácticas educativas realizadas en la EFCh. Ala vez, las universidades deben hacerse eco de los resultados a los que se ha llegado, pues ello podría servir para que la formación de profesores y profesoras se aleje de esa visión tecnocrática de la asignatura. Sobre todo ahora, pues Chile se encuentra en un momento de revisión, análisis y reconstrucción de los principios que sustentan su sistema educativo en cada uno de los niveles de la enseñanza formal.

Por último, es relevante considerar los nuevos retos que se señalan para la investigación en el área curricular que nos ocupa. Es desde una mayor y mejor comprensión de las prácticas educativas, la producción científica que se está realizando y el posicionamiento social de la asignatura, que se podrá entregar información valiosa para una reconfiguración de las políticas públicas relacionadas con la EF. Es por ello que estamos desarrollando algunas líneas de investigación que intentan dar respuesta a los retos señalados. Por un lado estamos trabajando en la construcción histórica de la asignatura EF desde el análisis de la publicación periódica más antigua que existe en el país (Revista Educación Física). A la vez estamos comenzando una investigación sobre la calidad de las prácticas corporales en la educación infantil. Por último, estamos elaborando un proyecto de investigación que analizará las prácticas corporales emergentes en el entramado de la ciudad. Ello porque muchas de esas prácticas son las que muestran mayor interés para los jóvenes, y la perspectiva deportivizada de la asignatura no les está dando cabida dentro del espacio-tiempo escolar.

\section{Agradecimientos}

Agradecemos al Fondo Nacional de Investigación Científica y Tecnológica de Chile(FONDECYT) por el financiamiento de la investigación FONDECYT Nº 11110016 titulada «Educación Física y su función de transformación de las desigualdades sociales: profesorado del área y documentación ministerial». Contexto desde el que se construyen el artículo precedente.

\section{Referencias}

ACE. (2013). Sistema de medición de la calidad de la educación. Santiago de Chile: Gobierno de Chile.

Almonacid, A., \& Matus, M. (2012). Una indagación al imaginario escolar desde el aula de Educación Física. Revista Educación Física y Deporte, 31(1), 791-805.

Blandón, M; Molina, V., \& Vergara, E. (2005). Los estilos directivos y la violencia escolar. Las prácticas de la educación física. Revista iberoamericana de educación, 38,87-103.

Canales, M. (2006). Metodologías de investigación social. Introducción a los oficios. Santiago de Chile: Editorial LOM.

Cárcamo, J. (2012). El profesor de educación física desde la perspectiva de los escolares. Estudios Pedagógicos, 38(1), 105-119.

CONICYT. (2005-2012). Panorama científico. Santiago de Chile: Gobierno de Chile.

Córdoba, T. (2011). Educación física e inclusión del alumnado con discapacidad. Sobre ruedas, 77, 14-15.

CPEIP. (2013). Estándares para la formación inicial de los profesores de educación física y salud. Santiago de Chile: Gobierno de Chile.

Daroch, V. (2010). Percepción de prestigio y valorización social de profesión docente,Una mirada desde los profesores de colegios municipales y profesores jubilados en el año 2010. Tesis para optar al título de socióloga. Satiago de Chile: Universidad de Chile.

Decreto $N^{\circ} 2960 / 2012$ por el que se aprueban planes y programas de estudios de educación básica en cursos y asignaturas que indica. Recuperado de http://bcn.cl/16sza

Delgado, E.; Ruiz, R. \& Jiménez, E. (2006). La edición de Revistas Científicas. Directrices, criterios y modelos de evaluación. Granada: Universidad de Granada.

Deutscher Sportbund (2006). Organisation unddurchführung des sportunterricht aus sicht der schulleitung. Westfalia: DSB-SPRINTStudie.

Escartí, A., Buelga, S., Gutiérrez, M., Pascual, C. (2009). El desarrollo positivo a través de la actividad física y el deporte: el programa de responsabilidad personal y social. Revista de Psicología General y Aplicada, 62(1-2), 45-52.

Escartí, A., Pascual, C. \& Gutiérrez, M. (2005). Responsabilidad personal y social a través de la educación física y el deporte. Barcelona: Graó.

Eurydice. (2013). La Educación Física y el deporte en los centros escolares de Europa. Madrid: Redie.

Flecha, R. \& Molina, S. (2013). Aportaciones del proyecto INCLUDED a la mejora de la gestión educativa. Organización y gestión educativa, 21(5), 26-27.

Flick, U. (2004). Introducción a la investigación cualitativa. Madrid: Editorial Morata.

Fox, K. (1991). Motivating children for physical activity: towards a healthier future. Journal of Physical Education, Recreation and Dance, 62, 34-38.

Gibbs, G. (2012). El análisis de datos cualitativos en investigación cualitativa. Madrid: Editorial Morata.

Giesecke, H. (1998). Pädagogische illusionen. Lehrenaus 30 Jahren Bildungspolitik. Stuttgart: Klett-Cotta.

Goetz, J. P. \& Lecompte, M. D. (1988). Etnografía y diseño cualitativo en investigación educativa. Madrid: Editorial Morata.

Gómez, I. \& Prat, M. (2009). Hacia una Educación Física crítica y transformadora de las prácticas sociales: de la práctica a la reflexión y viceversa.Cultura y Educación, 21(1), 67-88.

Hardman, K. (2008). Physical education and schools: a global perspective. Kinesiology, 40(1), 5-28.

Hardman, K. \& Marshall, J. (2000). Thestate and status of physical education in schools in international context. European Physical Education Review, 6(3), 203-229.

Kirk, D. (1990). Educación física y currículum. Introducción crítica. Valencia: Editorial Universidad de Valencia.

Kirk, D. (2006). Sport education, critical pedagogy, and learning theory: toward and intrinsic justification for physical education and youth sport. Quest, 58(2), 255-264.

Kirk, D. (2007). Con la escuela en el cuerpo, cuerpos escolarizados: la construcción de identidades inter / nacionales en la sociedad postdisciplinaria. Ágora para la Educación Física y el Deporte, 4-5, 3956.

Lenzen, D. (2000). Sport, bewegung order was? Argumentations rituale in der sport padagogik. Sportunterricht, 49, 77-80. 
Loaiza, M.; Sánchez, G. \& Villegas, G. (2004). Valoración económica del trabajo doméstico. Un abordaje desde el valor agregado en preparación de alimentos, en el aseo de la ropay de la casa. Caldas: Edumet.

López, V., García, J., García, A., González, M, López, E., Monjas, R., ... \& Archilla, M. (2005). ¿Cuándo tenemos poco prestigio? Mirando la viga en el ojo ajeno. Causas internas y reflexiones sobre el estatus de la educación física. Retos Nuevas Tendencias en Educación Física, Deporte y Recreación, 8, 11-18.

Lorente, E. (2005). Autogestión en educación física. Un estudio de caso en secundaria. Universidad de Barcelona. Tesis Doctoral. Recuperado de http://www.tdx.cat/TDX-1219105-084438

Mercedes, L. (2009). Educación física y discapacidad. Ágora para la Educación Física yel Deporte, 9, 7-14.

MINEDUC. (2013). Bases curriculares educación física y salud (Básica y Media) Santiago de Chile: Gobierno de Chile.

MINEDUC. (2013a). Bases curriculares educación física y salud. $1^{\circ} a$ $6^{\circ}$ Básico. Santiago de Chile: Gobierno de Chile.

Ministerio de Educación. (2011).Actuaciones educativas de éxito en las escuelas europeas. Madrid: Gobierno de España.

Ministerio Secretaría General de Gobierno (2013). Ministerio del Deporte. Ley $\mathrm{N}^{\circ} 20.686$ del 19 de agosto de 2013. Recuperado de http://bcn.cl/1fljp

MINSAL.(2009-2010). Encuesta nacional de salud. Santiago de Chile: Gobierno de Chile.

Moreno, A. (2011-2014). La educación física y su función de transformación de las desigualdades sociales: profesora del área y documentación ministerial. Proyecto Fondecyt Iniciación No 11110016. Santiago de Chile: CONICYT. Recuperado de https:// educacionfisicaytransformacionsocial.wordpress.com/

Moreno, A. (2014). El misterioso viaje de aprender a nadar, pensar y sentir: educación como proceso de creación de relaciones posibles. Polis, Revista Latinoamericana, 37, 1-14.

Moreno, A., Marlen, C., Almonacid,A., \& Vargas, A. (2013). La educación física chilena. Un modelo tecnocrático de la enseñanza y desvalorización del colectivo docente. Revista Tandem. Didáctica de la Educación Física, 42, 7-17.

Moreno, A. Gamboa, R. \& Poblete, C.(2014). La educación física en Chile: análisis crítico de la documentación ministerial.Revista Brasileña de Ciencias del Deporte, 36(2), 411-427.

Moreno, A.; Rivera, E. \& Trigueros, C.(2014). Sistema de medición de la calidad de la educación física Chilena.Revista Movimento,20(1), 145-167.
Pedraz, V. (2007). El cuerpo sin escuela: proyecto de supresión de la educación física escolar y qué hacer con su detritus. Ágora para la Educación Física y el Deporte, 4-5, 57-90.

Poblete, C. (2013).La construcción del conocimiento histórico de la EF en Chile: Una mirada la primera revista latinoamericana de la especialidad durante los años comprendidos entre 1934 y 1962.Tesis Doctoral. Granada: Universidad de Granada.

Poblete, C.; Moreno, A. \& Rivera, E. (2014). Educación Física en chile: Una historia de la disciplina en los escritos de la primera publicación oficial del Instituto de Educación Física de la Universidad de Chile(1934-1962). Revista Estudios Pedagógicos, 40(2), (en prensa)

Rodríguez, H. \& Flecha, R. (2011). La revolución científica y democrática en educación. Multidisciplinary Journal of Educational Research, 1(1), 34-52.

Santos, M. A. (2003). Trampas en educación: el discurso sobre la calidad. Madrid: Editorial Muralla.

Strauss, A. \& Corbin, J. (2002). Bases de la investigación cualitativa: Técnicas y procedimientos para desarrollar la teoría fundamentada. Colombia: Universidad de Antioquia.

Tenti, E. (2005). La condición docente: análisis comparado de la Argentina, Brasil, Perú, Uruguay. Buenos Aires: Editorial Siglo XXI.

Tirado, M. A. \& Ventura, C. (2009). Propuestas para el desarrollo de la competencia crítica en el alumnado de Educación Física. Culturay Educación, 21(1), 55-66.

Toro, S. (2007). Una aproximación epistemológica a la didáctica de la motricidad desde el discurso y práctica docente. Revista Estudios Pedagógicos, 33(1), 29-43.

Valenciano, J. (2007). Calidad y produccioìn cientiifica de las revistas espanbolas de ciencias de la actividad fiisica y el deporte. Tesis Doctoral. Valencia: Universidad de Valencia.

Valenciano, J.; Villamón, M. \& Devís, J. (2008). Evaluación y clasificación de las revistas científico-técnicas españolas de Ciencias de la Actividad Física y el Deporte. Revista Española de Documentación Científica, 31(3), 396-412.

Villamón, M.; Devís, J., \& Valenciano, J. (2005). Análisis de la visibilidad de las revistas científico-técnicas españolas de ciencias de la actividad física y el deporte. Revista de Psicología del Deporte, 14(2), 253-267.

Villamón, M.; Devís, J. \& Valenciano, J. (2006). Análisis de las instrucciones para autores de las Revistas Españolas de Ciencias de la Actividad Física y el Deporte. Motricidad: European Journal Of Human Movement, 16, 133-150.

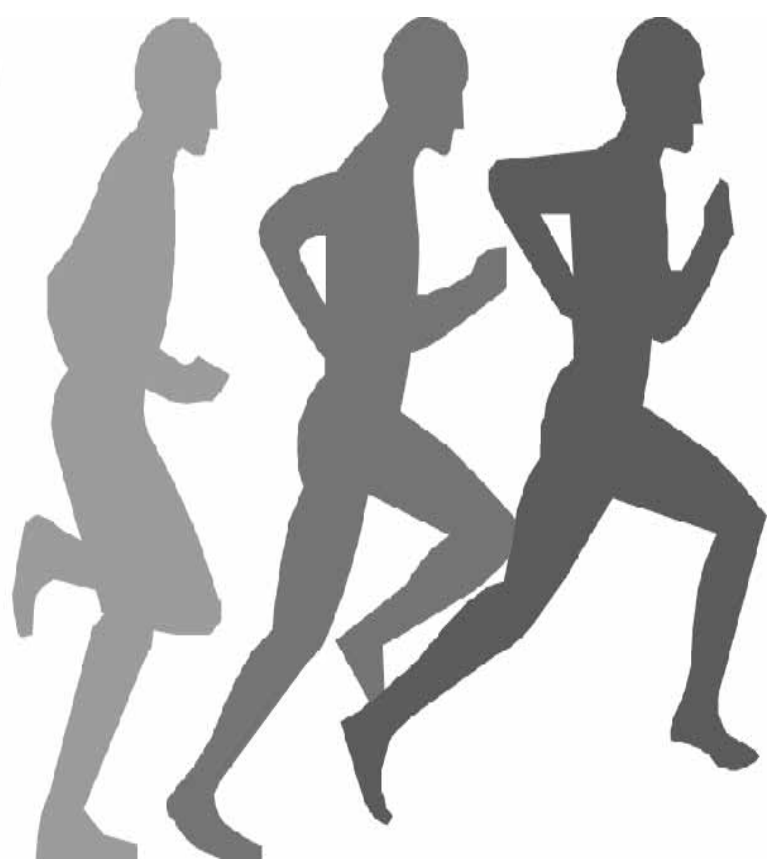

